\title{
QUÍMICA DE SEDIMENTO E ESTRUTURA DE BOSQUE EM MANGUEZAIS DO LITORAL DO PARANÁ
}

\author{
Sediment chemistry and forest structure in manguezais of the coast of paraná
}

Luís Fernando Roveda ${ }^{1}$, Thiago Vinícius Trento Occhi², Willian Thomaz Peçanha ${ }^{3}$, Cassiana Baptista Metri ${ }^{4}$, Rafael Metri ${ }^{5}$

\begin{abstract}
${ }^{1}$ Função; Professor Adjunto no Departamento de Ciências Biológicas, Universidade Estadual do Paraná - Campus Paranaguá; lfroveda@gmail.com (Autor para correspondências)

2Função; Doutorando em Ecologia e Conservação, Universidade Federal do Paraná; thiago.v.t.occhi@gmail.com

${ }^{3}$ Função; Doutorando em Genética e Biologia Molecular, Universidade Federal do Rio Grande do Sul; lila_thomaz@hotmail.com

${ }^{4}$ Função; Professor Adjunto no Departamento de Ciências Biológicas, Universidade Estadual do Paraná - Campus Paranaguá; cassiana.metri@unespar.edu.br

5Função; Professor Adjunto no Departamento de Ciências Biológicas, Universidade Estadual do Paraná - Campus Paranaguá; rmetri@yahoo.com.br
\end{abstract}

\author{
Artigo enviado em 27/03/2017, aceito em 12/06/2017 e publicado em 29/09/2017.
}

Resumo: As características químicas do sedimento em conjunto com a estrutura de bosques de mangue são informações fundamentais para compreender a dinâmica deste ambiente. O objetivo foi analisar parâmetros químicos e a estrutura de bosque em manguezais com diferentes graus de pressão antrópica. Foram estudados dois manguezais em Paranaguá, PR, um localizado próximo a um complexo industrial e ao porto de Paranaguá, e outro localizado na Unidade de Conservação na Floresta Estadual do Palmito. Foram avaliados os teores de fósforo, matéria orgânica, amônio, nitrato e nitrogênio disponível, assim como a densidade e perímetro a altura do peito das espécies Rižhophorae mangle, Laguncularia racemosa e Aviscenia schaueriana. O manguezal da Vila Becker, com maior interferência antrópica, teve maiores concentrações de fósforo, amônio e nitrogênio total, indicando ser um sedimento com melhor fertilidade. Não houve diferenças entre os manguezais com relação a estrutura de bosque, mas a Rizophorae mangle teve a maior densidade entre as espécies avaliadas, independente do manguezal. A proximidade destes ambientes ao meio urbano e industrial pode estar alterando as características químicas do sedimento o que futuramente pode alterar a estrutura de bosque destes manguezais.

Palavras-chave: Antropização; nutrientes; vegetação; estuário.

\begin{abstract}
The chemical characteristics in conjunction with the mangrove forest structure are key information to understand the dynamics of this environment. The objective was to analyze chemical parameters and forest structure in mangroves under different anthropogenic stresses. Two mangroves were studied in Paranaguá, PR, one located near an industrial complex and the port of Paranaguá, and another one located in the Conservation Unit in Palmito State Forest. The levels of phosphorus, organic matter, ammonium, nitrate and nitrogen available were evaluated as well as the density and perimeter of the breast height of the species Rizhophorae mangle, Laguncularia racemosa and Aviscenia schaueriana. The Vila Becker mangrove, with greater anthropogenic interference, had higher concentrations of phosphorus, ammonia and total nitrogen, indicating a sediment with better fertility. There were no differences among mangroves in relation to forest structure, but Rizophorae mangle had the highest density among the evaluated species, independent of the mangrove. The proximity of these environments to the urban and industrial environment may be altering the chemical characteristics of the sediment, which in the future may alter the forest structure of these mangroves.
\end{abstract}

Key Words: Anthropic pressure; nutrients; mangrove forest; 


\section{INTRODUÇÃO}

O ecossistema manguezal, predominante em regiões costeiras tropicais e subtropicais (ALVES;

NISHIDA, 2004), ocorre normalmente associado aos estuários, como ecossistema de transição entre o meio terrestre e o oceânico. $\mathrm{Na}$ costa brasileira, os manguezais ocupam originalmente cerca de $25.000 \quad \mathrm{~km}^{2}$ (SCHAEFFER-NOVELLI et al., 2000). No litoral do Paraná encontra-se um dos maiores estuários brasileiros, o Complexo Estuarino de Paranaguá (CEP), com 667 $\mathrm{km} 2$, predominantemente margeado por manguezais que cobrem cerca de $250 \mathrm{~km} 2$ (CASTELLA, 2006). A região abriga um intricado sistema composto por unidades de conservação, áreas urbanas e o maior porto do Sul do Brasil. Desta forma, evidencia-se constante ameaça para estes ecossistemas devido a ocupação urbana, as atividades industriais, o vazamento de produtos químicos, o desmatamento, a produção agrícola e a aquicultura (CHAKRABORTY et al., 2015).

Manguezais são ambientes de grande vulnerabilidade a ações antrópicas (SOUZA; VALE, 2016) especialmente considerando a ocorrência contínua e crescente de pressões nos ambientes costeiros em todo o mundo (MOLISANI et al., 2006; WEI, 2008; NOBI et al., 2010; BOAVENTURA et al., 2011). Segundo Copertino (2011) nos últimos 50 anos, entre $30 \%$ e $50 \%$ das florestas de mangues desapareceram e a taxa anual de perda deste ecossistema chega a ser 4 vezes a taxa de perda de florestas tropicais. Além da perda de áreas, vários descritores ambientais e biológicos podem ser alterados em locais antropizados.

Para Datta e Deb (2017) é visível a diferença de manguezais sob diferentes influencias antrópicas em variáveis como área basal, diversidade de espécies e densidade de árvores, bem como em características do solo como $\mathrm{pH}$, capacidade de troca cationnica, $\mathrm{Na} \%$ (sódio) permutável, C (carbono) total e estoques de C orgânico do sedimento. Percebe-se que tanto a estrutura dos bosques, como fatores abióticos do ecossistema podem servir como indicadores da qualidade ambiental.

A diversidade de espécies e a estrutura da vegetação também auxiliam na avaliação destes ecossistemas, visto estarem intimamente interligados (SOARES et al., 2003). Diferentes estudos demonstram que as espécies de mangue podem exibir dominância diferenciada em relação à condição ambiental, bem como variações no PAP (perímetro da altura do peito) e altura entre as espécies relacionados ao estado de conservação dos manguezais (SOARES et al., 2003; Silva et al., 2005; Madi et al., 2016).

A constituição química dos solos em manguezais é caracterizada pela variação natural, ligada a grande variabilidade e origem de materiais depositados nestes ecossistemas e também a fatores antropogênicos (CHRISTOPHORIDIS et al., 2009) que podem elevar os níveis de elementos químicos. Esta condição leva, consequentemente, a variações no crescimento de plantas se comparado com manguezais em regiões com pouca ou nenhuma interferência (KRAUSS et al., 2006). Desta forma, é evidente que ambos os fatores se complementam na caracterização dos ambientes.

Considerando que a caracterização química do sedimento e da estrutura de bosque estão intimamente ligados ao estado de conservação destes ambientes, sendo, assim, importantes para a compreensão da dinâmica deste ecossistema (BOAVENTURA et al., 2011), este estudo objetivou determinar estas condições em dois manguezais em Paranaguá-PR, avaliando a interferência antropogênica sobre o ecossistema manguezal.

\section{MATERIAIS E MÉTODOS}

O clima da região, segundo Köppen, é classificado como Cfa, ou seja, Clima Temperado, úmido e com temperaturas médias no mês mais quente acima dos $22^{\circ} \mathrm{C}$, não há existência de uma estação seca definida, ocorrendo precipitação em todos os meses do ano. O trabalho foi realizado em Paranaguá Paraná/BR em manguezais da CEP, sendo um com forte influência antrópica o manguezal da Vila Becker (MVB) $\left(25^{\circ}-50^{\prime} \mathrm{S}\right.$ e $\left.48^{\circ} 54^{\prime} \mathrm{W}\right)$, e outro preservado, localizado no manguezal da Floresta Estadual do Palmito (MFP) (25 $35^{\prime} \mathrm{S}$ e $\left.48^{\circ} 30^{\prime} \mathrm{W}\right)$, que por sua vez trata-se de uma Unidade de Conservação do Instituto Ambiental do Paraná (IAP).

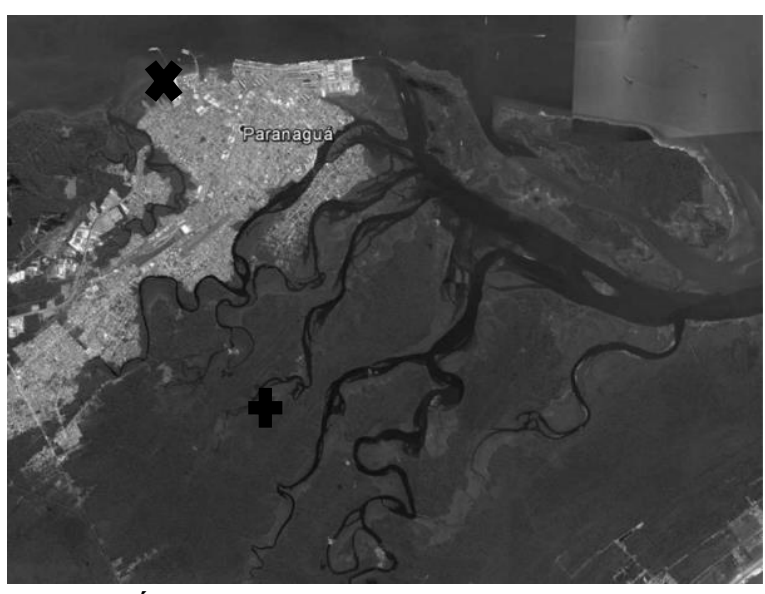

Figura 1: Áreas das coletas. "X" Manguezal da Vila Beker (MVB) e “+” Manguezal da Floresta Estadual do Palmito (MFP).

Fonte: Google Earth.

As coletas de amostras foram realizadas em maio e agosto de 2011. A área amostral (cada manguezal) foi dividida paralelamente à água em três regiões: infralitoral, médiolitoral e supralitoral (estas divisões estavam relacionadas com a altura do dossel das plantas). 
Cada região foi subdividida aleatoriamente em quatro parcelas experimentais com área de $600 \mathrm{~m}^{2}$ cada (paralelas ao rio), entre parcelas foi ainda deixado uma área como margem de segurança. A região do infralitoral iniciava ao lado do rio, a mesolitoral iniciava a uma distância de $30 \mathrm{~m}$ do rio e a supra litoral a uma distância de $60 \mathrm{~m}$ do rio.

O sedimento foi coletado com auxílio de uma pá de jardineiro. Foram coletadas três sub amostras de sedimento de aproximadamente $100 \mathrm{~g}$ em cada parcela, as mesmas foram homogeneizadas formando uma única amostra com aproximadamente $300 \mathrm{~g}$, as quais foram ensacadas, identificadas e encaminhadas ao laboratório de Ecologia Ambiental da Universidade Estadual do Paraná, campus Paranaguá. Em laboratório, as amostras de sedimento foram secas em estufa a $65^{\circ} \mathrm{C}$ por 24 horas até peso constante. Posteriormente, as amostras foram peneiradas em malha de $2 \mathrm{~mm}$ e armazenadas para análise. Os parâmetros avaliados foram os teores de fósforo $(\mathrm{P})$, matéria orgânica (MO), amônio (AM), nitrato (NT) e nitrogênio total (NT - soma do Amônio + Nitrato). O P foi determinado por extração com solução duplo-ácida ( $\mathrm{HCl} 0,05 \mathrm{M}+\mathrm{H}_{2} \mathrm{SO}_{4}$ 0,0125 M) e posterior leitura em espectrofotômetro. A MO foi quantificada por meio do método volumétrico por dicromatro de potássio e titulação do excesso de dicromato de potássio pelo sulfato ferroso amoniacal conforme descrito em Silva (20019). A AM, o NT e o ND foram determinados através da destilação por arraste de vapores, condensação e depositação em solução de ácido bórico. A quantificação do $\mathrm{N}$ nas formas de AM e NT se deu por titulação do $\mathrm{H}_{2} \mathrm{SO}_{4} 0,005$ $\mathrm{M}$, via restituição do ácido bórico, conforme descrição de SILVA (2009). Foi mensurado ainda o pH com auxílio de pHmetro de bancada conforme metodologia descrita em Silva (2009).

A fim de coletar dados para análise estrutural da vegetação, foram alocadas três sub parcelas, demarcadas aleatoriamente, cada uma com área de 20 $\mathrm{m}^{2}$. Todos os espécimes com altura superior a $1 \mathrm{~m}$ foram identificadas por meio do APG III e contabilizadas para análise de densidade. Após foram coletadas as medidas referentes ao perímetro à altura do peito $(\mathrm{PAP})(1,30 \mathrm{~m})$ com fita métrica.

Os dados foram submetidos à análise de variância através de um delineamento inteiramente cazualizado em esquema fatorial $2 \times 3$ (2 manguezais $\times 3$ zonas) para os resultados da avaliação química dos sedimentos e um delineamento fatorial $2 \times 3 \times 3 \quad(2$ manguezais $\times 3$ zonas $\times 3$ espécies) para os resultados da avaliação das plantas. Quando constatada significância, as médias foram comparadas com o teste de Tukey ao nível de $5 \%$ de erro com auxílio do programa ASSISTAT versão 7.6 Beta.

\section{RESULTADOS E DISCUSSÃO}

Conforme os valores dos parâmetros químicos do sedimento (Tabela 1) dos manguezais estudados, observou-se uma distinção entre valores e distribuição de P do sedimento. No manguezal da Vila Becker (MVB) foi observado teor de $\mathrm{P}$ superior ao do manguezal da Floresta Estadual do Palmito (MFP), demonstrando teor de $\mathrm{P}$ crescente no sentido infralitoral para o supralitoral. Entretanto, padrão inverso foi observado no MFP, com teores de $\mathrm{P}$ decrescentes no sentido infralitoral para o supralitoral.

No CEP, há tendência das planícies entre marés próximas à desembocadura terem maiores taxas de inundação, devido a menor declividade do terreno e a inundação ocorrer logo após a preamar nessas áreas (ALVES, 2011), o que pode favorecer a maior concentração de P no MFP devido a sua proximidade com a abertura do estuário. A distribuição de $\mathrm{P}$ no MFP está de acordo com o observado na literatura (KRAUSS et al. 2006). No estuário de São Matheus-ES, os valores de fósforo foram maiores em áreas próximas à desembocadura e diminuíram nas estações secas (BERNINI et al. 2010). Segundo Madi, Boeger e Reissmann (2015), em manguezais do litoral do Paraná os teores de $\mathrm{P}$ em sedimentos variaram entre 33 a $36 \mathrm{mg}$ $\left.\mathrm{dm}^{-3}\right)$.

No MVB o fósforo tem concentrações 40 vezes superiores ao do MVP no supralitoral, 11 vezes superior no meso e igual no infralitoral, indicando um sentido de deposição contrário em relação ao MFP. Estes resultados sugerem influência antrópica no local o que está relacionado a presença de atividade industrial, pois próximo ao supralitoral do MFP estão instaladas empresas que armazenam e transportam fertilizantes de uso na agricultura. Portanto, resíduos de fertilizantes devem ser perdidos durante o transporte na área industrial e acabam levados pelas chuvas e depositados no manguezal, elevando a concentração de P. Os teores de MO não diferiram significativamente entre os manguezais estudados (Tabela 1). Porém, na média dos manguezais, o teor de $\mathrm{MO}$ foi maior na região mesolitoral em comparação a supralitoral. A região do infralitoral fica mais próxima da água, o que pode indicar um ambiente com maior anoxia que regiões mais distantes como a supralitoral, assim pode ocorrer maior oxidação nessa região e, com isso, maior mineralização e menor acúmulo da MO no sedimento (Wilson et al., 2012). Ainda, segundo Sakho et al. (2014), a maior concentração de Rižphora mangle permite uma menor oxigenação do sedimento via sistema radicular, em comparação a outras espécies presentes, o que também favorece a baixa degradação da MO no sedimento. Entretanto, um estudo no estuário de São Matheus-ES, mostrou teores de $\mathrm{MO}$ superiores em regiões distantes da desembocadura (SILVA; BERNINI e CARMO, 2005; BERNINI et al., 2006), mostrando uma variação 
Tabela 1 - Teores médios de fósforo (P), matéria orgânica (MO), amônio, nitrato, nitrogênio total (soma amônio + nitrato) e $\mathrm{pH}$ do sedimento avaliados no infralitoral (Infra), mesolitoral (Meso) e supralitoral (Supra) no manguezal localizado na Floresta Estadual do Palmito (MFP) e no manguezal localizado na Vila Becker (MVB).

\begin{tabular}{|c|c|c|c|c|}
\hline Manguezais/região & Infra & Meso & Supra & Média \\
\hline \multicolumn{5}{|c|}{$\mathrm{P}\left(\mathrm{mg} \mathrm{dm}^{-3}\right)$} \\
\hline MFP & $17,0 \mathrm{aA}$ & $6,3 \mathrm{bB}$ & $4,5 \mathrm{bB}$ & $9,29 \mathrm{~b}$ \\
\hline MVB & $19,8 \mathrm{aC}$ & $70,1 \mathrm{aB}$ & $180,9 \mathrm{aA}$ & 90,34 a \\
\hline Média & $18,4 \mathrm{~B}$ & $38,2 \mathrm{~B}$ & $92,7 \mathrm{~A}$ & \\
\hline \multicolumn{5}{|c|}{$\mathrm{MO}\left(\mathrm{g} \mathrm{Kg}^{-1}\right)$} \\
\hline MFP & 14,0 & 15,8 & 10,3 & $13,4 \mathrm{a}$ \\
\hline MVB & 15,0 & 33,5 & 13,3 & $20,6 \mathrm{a}$ \\
\hline Média & $14,5 \mathrm{AB}$ & $24,7 \mathrm{~A}$ & $11,8 \mathrm{~B}$ & \\
\hline \multicolumn{5}{|c|}{$\mathrm{pH}$} \\
\hline MFP & 5,8 & 5,9 & 5,9 & $5,9 \mathrm{~b}$ \\
\hline MVB & 6,2 & 6,04 & 6,7 & $6,3 \mathrm{a}$ \\
\hline Média & 6,0 & 5,9 & 6,3 & \\
\hline \multicolumn{5}{|c|}{ Amônio (mg kg-1) } \\
\hline MFP & 9,32 & 12,25 & 10,5 & $10,69 \mathrm{~b}$ \\
\hline MVB & 22,17 & 20,7 & 22,3 & $21,72 \mathrm{a}$ \\
\hline Média & 15,75 & 16,47 & 16,4 & \\
\hline \multicolumn{5}{|c|}{ Nitrato $\left(\mathrm{mg} \mathrm{kg}^{-1}\right)$} \\
\hline MFP & 11,1 & 11,6 & 11,6 & 11,4 \\
\hline MVB & 9,3 & 10,5 & 10,4 & 10,1 \\
\hline Média & 10,2 & 11 & 11 & \\
\hline \multicolumn{5}{|c|}{ Nitrogênio total $\left(\mathrm{mg} \mathrm{kg}^{-1}\right)$} \\
\hline MFP & 20,42 & 23,92 & 22,17 & $22,17 \mathrm{~b}$ \\
\hline MVB & 31,5 & 31,2 & 32,8 & $31,83 \mathrm{a}$ \\
\hline Média & 25,96 & 27,56 & 27,48 & \\
\hline
\end{tabular}

Letras diferentes diferem estatisticamente ao nível de 5\% pelo teste de Tukey. Letras maiúsculas para linha e minúscula para coluna.

espacial diferenciada do presente estudo. A variação do teor de MO está sujeita ao regime de marés e a produção de serrapilheira no local, podendo ser distinto de acordo com o manguezal (SILVA; BERNINI e CARMO, 2005; BERNINE et al., 2006).

Os manguezais tiveram $\mathrm{pH}$ levemente ácido, sendo maior no MVB (Tabela 1). Não foram observadas diferenças de $\mathrm{pH}$ nas regiões dos dois manguezais estudados. $\mathrm{O}$ maior $\mathrm{pH}$ observado no MVB pode estar relacionado a maior proximidade de águas marinhas, visto seu $\mathrm{pH}$ ser superior em comparação a águas com menores concentrações de sais (SILVA; BERNINI e CARMO, 2005). Assim como a MO, o pH está sujeito a mudanças com as variações de marés e com o regime pluviométrico (CUZZUOL; CAMPOS, 2001), entretanto estudos apontam que o $\mathrm{pH}$ em manguezais varia de levemente ácido a neutro (CUZZOUL; CAMPUS, 2001; BERNINI et al., 2006), assim como observado no presente estudo.

Os teores de AM e ND diferiram entre os manguezais, com maiores teores no MVB, enquanto o teor de NT não diferiu entre os manguezais ou entre as regiões analisadas (Tabela 1). O aumento dos teores de AM e ND no MVB estão relacionados a deposição de efluentes urbanos e industriais no local, que pode ser constatado visualmente nos arredores. Estas deposições acarretam na eutrofização do ambiente aquático, o que vai favorecer a proliferação de algas, consequentemente aumentando turbidez da água acarretando na alteração da dinâmica do ambiente aquático (EMBRAPA, 2002). 
Tabela 2 - Densidade média de Rizophora mangle (RM), Laguncularia racemosa (LR) e Avicenia schaueriana (AS) no infralitoral (Infra), mesolitoral (Meso) e Supralitoral (Supra) no manguezal da Floresta Estadual do Palmito (MFP) e no manguezal localizado na Vila Becker (MVB).

\begin{tabular}{|c|c|c|c|c|c|}
\hline \multirow{2}{*}{ Parâmetros analisados } & & \multicolumn{4}{|c|}{ Espécies (para cada $10 \mathrm{~m}^{2}$ ) } \\
\hline & & $\mathrm{RM}$ & LR & AS & Média \\
\hline \multirow{3}{*}{ Densidade de Espécie } & Infra & 0,53 & 0,04 & 0,05 & 0,20 \\
\hline & Meso & 0,86 & 0,02 & 0,005 & 0,3 \\
\hline & Supra & 0,49 & 0,32 & 0,34 & 0,39 \\
\hline Média Espécie & & $0,63 \mathrm{~A}$ & $0,13 \mathrm{~B}$ & $0,13 \mathrm{~B}$ & \\
\hline MFP & & $0,52 \mathrm{aA}$ & $0,008 \mathrm{aB}$ & $0,26 \mathrm{aAB}$ & 0,26 \\
\hline MVB & & $0,74 \mathrm{aA}$ & $0,25 \mathrm{aB}$ & $0,008 \mathrm{aB}$ & 0,33 \\
\hline
\end{tabular}

Letras diferentes diferem estatisticamente ao nível de $5 \%$ pelo teste de Tukey. Letras maiúsculas para linha e minúscula para coluna.

Em geral não houve diferença significativa entre a densidade de plantas nos manguezais estudados (Tabela 2). Em ambos os ambientes houve a ocorrência das três espécies típicas de manguezais, em ordem de dominância: Rižphora mangle, Laguncularia racemosa e Avicennia schaueriana. Porém para os dois manguezais houve o predomínio de R. mangle. Madi et al. (2016), também constataram a presença dessas três espécies estudando manguezais do litoral do Paraná, porém observaram dominância de diferentes das espécies. Os manguezais também não diferiram em relação a distribuição das espécies ao longo do gradiente de influência da maré. No MFP a densidade de R. mangle foi superior em todos os extratos, os mesmos resultados também foram observados no MVB.

Considerando a densidade total independentemente do manguezal, o gênero Rizophora teve dominância em relação aos demais, indicando valores médios em ambos os manguezais de 630 indivíduos/ha, o que indica manguezais com maior grau de maturidade. Esse resultado corrobora os valores encontrados por Silva, Bernini e Carmo (2005), que estudaram o estuário de São Matheus-ES, onde foram observadas densidades de 450 a 1450 indivíduos/ha.Em contrapartida, Martins, Couto e Delabie (2011) observaram densidades médias acima de 1450 indivíduos ha-1, e Silva et al. (2016) observaram densidade acima de 5000 indivíduos ha ${ }^{-1}$. A densidade do bosque está relacionada a sua maturidade e desenvolvimento. Em bosques mais jovens é observada maior densidade e menores perímetros de plantas, enquanto em bosques mais velhos ocorre menor número de plantas com perímetros maiores (SCHAEFFER-NOVELLI et al., 2000; MARTINS; COUTO; DELABIE, 2011).

A espécie R. mangle teve o maior PAP dentre as espécies nos manguezas avaliados (Tabela 3). Entre os manguezais, foram observadas diferenças no PAP das plantas em relação a $R$. mangle e a $A$. schaueriana, indicando maiores perímetros para ambas as espécies no MFP. Isso indica que a característica química distinta dos locais pode ter influenciado o desenvolvimento das plantas, porém o maior PAP e, portanto, o maior desenvolvimento ocorreu em condições naturais e sem o aporte de nutrientes de fonte antropogênica. $O$ fato do MVB estar mais perto da foz (montante) aumenta as concentrações de sais no ambiente. Segundo Martins, Couto e Delabie (2011), estes bosques podem ser mais jovens que os mais distantes, o que também poderia explicar estas diferenças, já que a densidade pouco

Tabela 3 - Perímetro médio na altura do peito (PAP) de Rizophora mangle (RM), Laguncularia racemosa (LR) e Avicenia schaueriana (AS) no infralitoral (Infra), mesolitoral (Meso) e Supralitoral (Supra) no manguezal da Floresta Estadual do Palmito (MFP) e no manguezal localizado na Vila Becker (MVB).

\begin{tabular}{cccccc}
\hline \multicolumn{2}{c}{ Parâmetros analisados } & \multicolumn{2}{c}{ PAP de Espécies (cm) } \\
& & RM & LR & AS & Média \\
\hline \multirow{2}{*}{$\begin{array}{c}\text { Perímetro médio } \\
\text { das Espécie }\end{array}$} & Infra & 20,0 & 0 & 19,0 & 15,3 \\
\multicolumn{2}{c}{ Média Espécie } & 16,9 & 8,2 & 5,9 & 10,2 \\
\multicolumn{2}{c}{ MFP } & 13,4 & 2,0 & 13,2 & 9,5 \\
\multicolumn{2}{c}{ MVB } & $16,8 \mathrm{~A}$ & $4,5 \mathrm{~B}$ & $9,9 \mathrm{~B}$ & $16,0 \mathrm{a}$ \\
\hline
\end{tabular}

Letras diferentes diferem estatisticamente ao nível de $5 \%$ pelo teste de Tukey. Letras maiúsculas para linha e minúscula para coluna. 
influenciou estes valores já que ambas as áreas apresentaram valores muito próximos, o que pode indicar bosques de mesma idade. No estudo de Silva et al. (2016), contudo, maior desenvolvimento estrutural foi observado nas zonas mais distantes da planície hipersalina. Assim, as altas concentrações de $\mathrm{P}$ e $\mathrm{N}$ podem estar influenciando de forma negativa $\mathrm{O}$ desenvolvimento das espécies vegetais, podendo principalmente no caso do $\mathrm{P}$ estar causando uma fitotoxidez as plantas, já que suas concentrações no MVB chegaram a ficar quase $40 \mathrm{x}$ superiores $\mathrm{em}$ comparação ao MFP, e, assim, diminuindo seu desenvolvimento. Os valores médios de DAP observados neste estudo corroboram os valores observados por outros autores que determinaram o perímetro de altura do peito, com intuito de avaliar a estrutura dos bosques de mangue e de diferentes espécies, (SOARES et al., 2003; BERNINI; REZENDE, 2004; SILVA; BERNINI e CARMO, 2005; BERNINE et al. 2006; BERNINI; RESENDE, 2009; SALES et al. 2009).

\section{CONCLUSÕES}

Os manguezais estudados apresentaram diferenças nos teores de nutrientes. O manguezal com maior proximidade de indústrias e do porto teve maiores teores de $\mathrm{P}$ e $\mathrm{N}$ no sedimento, confirmando a interferência antropogênica no local.

A R. mangle foi a espécie dominante em ambos os manguezais, bem como teve os maiores perímetros. Os maiores perímetros para $\mathrm{R}$. mangle e A. schaueriana foram observados no MFP.

Fatores antropogênicos podem estar influenciando de forma negativa o desenvolvimento das plantas no MVB.

Mais estudos são necessários para avaliar outros fatores antropogênicos de interferência que podem estar causando efeitos negativos a este ecossistema.

\section{REFERÊNCIAS}

ALVES, R. R. N.; NISHIDA A. K. Population structure of the mangrove crab Ucides cordatus (Crustacea: Decapoda ; Brachyura) in the estuary of the Mamanguape river, Northeast Brazil. Tropical Oceanography, v. 32, n. 1, p. 23-37, 2004.

ALVES, D. S. M. O Papel das taxas de inundação na distribuição da vegetação e fauna em manguezais de um estuário subtropical. 59 f. Dissertação (Mestrado em zoologia) Universidade Federal do Paraná, Curitiba, 2011.

BERNINI, E.; REZENDE, C. E. Estrututa da vegetação em florestas de mangue do estuário do rio
Paraíba do Sul, estado do Rio de Janeiro, Brasil. Acta Botânica Brasilica, v. 18, n.3, p. 491-502, 2004.

BERNINI, E.; DA SILVA, M. A. B.; DO CARMO, T. M. S.; CUZZOUL, G. R. F. Composição química do sedimento e de folhas das espécies do manguezal do estuário do rio São Mateus, Espírito Santo, Brasil. Revista Brasileira de Botânica, v.29, n.4, p. 689-699, 2006.

BERNINI, E.; REZENDE, C. E. Variação estrutural em florestas de mangue do estuário do rio Itabapoana, ES-RJ. Biotemas, v. 23, n.1, p.49-60, 2009.

BERNINI, E.; DA SILVA, M. A. B.; DO CARMO, T. M. S.; CUZZOUL, G. R. F. Spatial and temporal variation of the nutrients in the sediment and leaves of two Brazilian mangrove species and their role in the retention of environmental heavy metals. Brazilian society of plant physiology, v. 22, n.3, p.177-187, 2010.

BOAVENTURA, S. F.; HADLICH, G. M.; CELINO, J. J. Índices de contaminação de metais traço em encostas, manguezais e apicuns, Madre de Deus, Bahia. Geociências, v. 30, n. 4, p. 631-639, 2011.

CASTELLA RMB, CASTELLA PR, FIGUEIREDO DCS, QUEIROZ SMP (Orgs.) (2006) Mar e Costa: Subsídios para o ordenamento das áreas estuarina e costeira do Paraná. SEMA. Curitiba.

COPERTINO, M. da S., MARGARETH. Add coastal vegetation to the climate critical list. Nature, v. 473, n. 7347, p. 255, 2011.

CUZZUOL, G. R. F.; CAMPOS, A. Aspectos nutricionais na vegetação de manguezal do estuário do rio Mucuri, Bahia, Brasil. Revista Brasileira de Botânica, v.24, n.2, p.227-234, 2001.

DATTA, D., DEB, S. Forest structure and soil properties of mangrove ecosystems under different management scenarios: Experiences from the intensely humanized landscape of Indian Sunderbans. Ocean \& Coastal Management, v. 140, p. 22-33, 2017.

KRAUSS, K. W.; DOYLE, T. W.; TWILLEY, R. R.; RIVERA-MONROY, V. H.; SULLIVAN, J. K. Evaluating the relative contribution of hydroperiod and soil fertility on growth of south Florida mangrove. Hydrobiologia, v. 569, p. 311-324, 2006.

MADI, A. P. L. M., BOEGER, M. R. T., REISSMANN, C. B. Composição química do solo e das folhas e eficiência do uso de nutrientes por espécies de manguezal. Revista Brasileira De Engenharia Agricola e Ambiental, v. 19, n. 5, p. 433-438, 2015. 
MADI, A. P. L. M., BOEGER, M. R. T., LARCHER, L., PELOZO, A., SERENESKI, C., REISSMANN, C. B., PADIAL, A. A. Estrutura do componente de regeneração natural e arbóreo de dois manguezais no estado do paraná. Ciência Florestal, v. 26, n. 1, p. 159-170, 2016.

MARTINS, P. T. A.; COUTO, E. C. G.; DELABIE, J. H. C. Fitossociologia e estrutura vegetal do manguezal do rio Cururupe (Ilhéus, Bahia, Brasil). Gestão costeira integrada, v. 11, n.2, p. 163-169, 2011.

MOLISANI, M.M.; KJERFVE, B.; SILVA, A.P.; LACERDA, L.D. Water discharge and sediment load to Sepetiba Bay from an anthropogenically-altered drainage basin, SE Brazil. Journal of Hydrology, v. 331, p. 425-433, 2006.

NOBI, E.P.; DILIPAN, E.; THANGARADJOU, T.; SIVAKUMAR, K.; KANNAN, L. Geochemical and geostatistical assessment of heavy metal concentration in the sediments of different coastal ecosystems of Andaman Islands, Índia. Estuarine, Coastal and Shelf Science, v. 87, p. 253-264, 2010.

REZENDE, A. V. Agricultura e qualidade da água: Contaminação da água por nitrato. Planaltina: EMBRAPA, 2002. v. 1, 28p.

SAKHO, I., MESNAGE, V., COPARD, Y., DELOFFRE, J., FAYE, G., LAFITE, R., NIANG, I., A crosssection analysis of sedimentary organic matter in a mangrove ecosystem under dry climate conditions: The Somone Estuary, Senegal, African Earth Sciences, v. 101, p. 220-231, 2014.

SALES, J. B. L.; MEHLIG, U.; NASCIMENTO J. R.; FILHO, L. F. R.; MENEZES, M. P. M. Análise estrutural de dois bosques de mangue do rio Cajatuba, município de Marapanim, Pará, Brasil. Bol. Mus. Para. Emílio Goeldi. Ciências Naturais, v. 4, n. 1, p. 27-35, 2009.
SCHAEFFER-NOVELLI, Y. ; CONTRÓNMOLERO, G. ; SOARES, M. L. G, DE-ROSA, T. Brazillian Mangroves. Aquatic Ecosystem Helth \& Management, v. 3, p 561 - 570, 2000.

SILVA, F. C. da. Manual de análises químicas de solos, plantas e fertilizantes. - 2. ed. rev. ampl. - Brasília, DF : Embrapa Informação Tecnológica, 2009. 627 p.

SILVA, S. F. L DA, SANTOS, M. A. DOS, LAGEPINTO, F., BERNINI, B. Fitossociologia de uma floresta de mangue adjacente a uma planície hipersalina no estuário do Rio Miriri, Paraíba, Brasil. Revista Nordestina de Biologia, v. 24, n. 2, p. 3-12, 2016.

SILVA, M. A. B.; BERNINI, E.; CARMO, T. M. S. Características estruturais de bosques de mangue do estuário do rio São Matheus, ES, Brasil. Acta Botânica Brasilica, v.19, n.3, p. 465-471, 2005.

SOARES, M. L. G.; CHAVES, F. O.; CORRÊA, M. F.; JÚNIOR, M. G. S. Diversidade estrutural de bosques de mangue e sua relação com distúrbios de origem antrópica: o caso da baía de Guanabara (Rio de Janeiro). Anuário do Instituto de Geociências, v. 26, p.101-116, 2003.

SOUZA, S. O., VALE, C. C. do. Vulnerabilidade ambiental da planície costeira de Caravelas (BA) como subsídio ao ordenamento ambiental. Sociedade \& Natureza, v. 28, n. 1, p. 147-159, 2016.

SOUZA, T. de A. B.; CLEMENTE, G.; MOURA, F.; GARCIA, F.; FLYNN, M. Mapeamento de Manguezal em Cananéia, São Paulo - Brasil. In: Environmental and Health World Congress, 2006, Santos.

EI, M.; YANWEN, Q.; BINGHUI, Z.; LEI, Z. Heavy metal pollution in Tianjin Bohai Bay, China. Journal of Environmental Sciences, v. 20, p. 814-819, 2008. 